(2) Open Access Full Text Article

\title{
Pseudomyxoma extraperitonei in horseshoe kidney masquerading as renal hilar tumor: a case report
}

\author{
Xin Wang* \\ Xiang Gao* \\ Liang Wang \\ Zhihong Dai \\ Bo Fan \\ Haoyu Cui \\ Zhiyu Liu
}

Department of Urology, Second University, Dalian, Liaoning, People's Republic of China

*These authors contributed equally to this work Affiliated Hospital of Dalian Medical

This article was published in the following Dove Press journal: OncoTargets and Therapy

\begin{abstract}
Pseudomyxoma peritonei, a rare condition consisting of intraperitoneal mucinous tumors and ascites, most commonly arises from mucinous tumors of the appendix. Very rarely, mucinous deposits arise in the retroperitoneum without intraperitoneal involvement. This has been termed pseudomyxoma extraperitonei. It is a rare and poorly understood condition that is heterogeneous in its clinical behavior, and only a few cases presenting as localized disease in the retroperitoneum have been reported. In this paper, we report the first case of pseudomyxoma extraperitonei presenting as a simple renal hilar mass and mimicking a tumor of renal origin in a horseshoe-kidney patient. The patient underwent isthmusectomy and nephrectomy. Immunohistochemical staining suggested appendiceal origin. She remained alive without adjuvant therapy postoperatively, and no evidence of recurrence was present for 25 months.

Keywords: pseudomyxoma extraperitonei, pseudomyxoma peritonei, horseshoe kidney, renal hilar tumor
\end{abstract}

\section{Introduction}

Pseudomyxoma peritonei (PMP) is a rare neoplastic condition that is characterized by disseminated intraperitoneal mucinous tumors, often with gelatinous ascites in the abdomen or pelvis, and usually secondary to an appendiceal mucinous tumor. ${ }^{1}$ Occasionally, mucinous tumors in other sites, such as the colon, ovaries, pancreas, and urachus, are the culprits. ${ }^{2}$ The entity of pseudomyxoma extraperitonei (PME) in which mucinous implants arise in the retroperitoneum without any intraperitoneal involvement is even more uncommon. Because the biological behavior of PMP has been plagued with controversy and confusing terminology, this condition poses challenges in diagnosis and management. ${ }^{3}$

Clinically, its manifestations vary depending on the origin and location of tumors, which usually present with a variety of unspecific signs and symptoms. ${ }^{3}$ We report here on a 52-year-old female horseshoe-kidney patient, the first documented, with PME presenting as a simple renal hilar mass who underwent isthmusectomy and nephrectomy. We also review the previous literature of PMP involving the kidney and investigate tumor-specific characteristics and possible therapeutic strategies, in order to be aware of the characteristics of the tumor in various clinical settings.

Department of Urology, Second Affiliated Hospital of Dalian Medical University, 467 Zhongshan Road, Dalian, Liaoning I I6027, People's Republic of China

Tel +86 4II 8467 I29|

Email Izydoct@I63.com

\section{Case report}

A 52-year-old woman consulted at our hospital for a 10-day history of progressive dull pain in her right flank on August 5, 2016. Her symptoms were unrelated to 

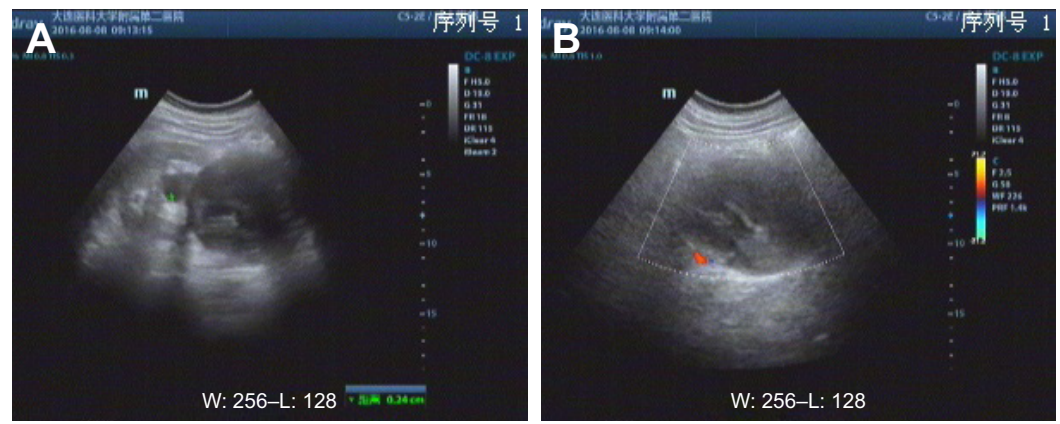

Figure I Ultrasonography of the right kidney.

Notes: (A) Giant complex cystic-solid mass $(10.1 \times 8.0 \mathrm{~cm})$ with inhomogeneous liquid dark area and high-echo stripes on the medial aspect of the right kidney; (B) colored blood-flow signals displayed on the margins of the mass, which was located close to the right kidney.

bowel movements, and she had no other gastrointestinal or urinary symptoms. On physical examination, a circle-like mass approximately $5 \mathrm{~cm}$ in diameter was discovered in the right upper abdominal quadrant and was tender to palpation. Right costovertebral angle tenderness was also present on percussion.

Laboratory tests revealed an elevated white-blood-cell count $\left(11 \times 10^{9} \mathrm{cells} / \mathrm{L}\right)$. All other hematological and biochemical tests, as well as urinalysis, were within normal limits.
The patient was admitted for a closer investigation of the right-flank mass. Ultrasonography revealed hydronephrosis caused by a well-circumscribed, hypoechogenic, complex mass measuring approximately $10.1 \times 8.0 \mathrm{~cm}$. The mass was located in the medial aspect of the right renal hilum and projected into the renal pelvis. All this was accompanied by an inhomogeneous liquid dark area and strong-echo stripes (Figure 1). Enhanced computed tomography (CT; Figure 2A and $\mathrm{B}$ ) and magnetic resonance imaging (Figure 3) scans
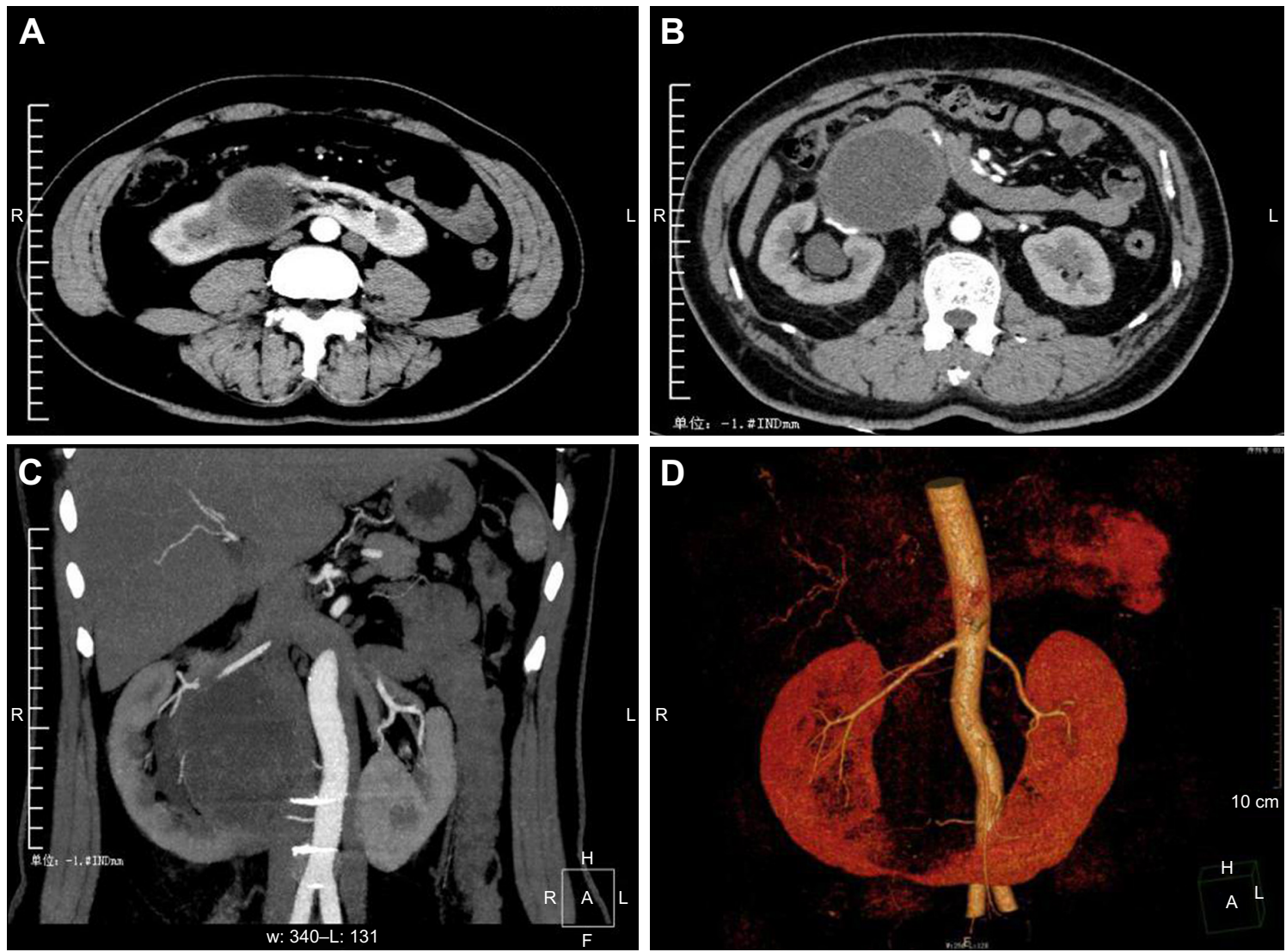

Figure 2 Enhanced computed tomography (CT) scan showing a cross-sectional view of the urinary system.

Notes: (A) Fusion of both renal lower poles and hydronephrosis due to a large, well-circumscribed mass located medial to the right renal hilum. (B) Spherical cystic-solid mass $(9.5 \times 8.7 \mathrm{~cm})$ of heterogeneous density with CT-attenuation values of 18-35 HU, likely arising from the anteromedial aspect of the right half of the horseshoe kidney. (C, D) Renal CT angiogram showing compression of the renal artery and narrow renal veins. 

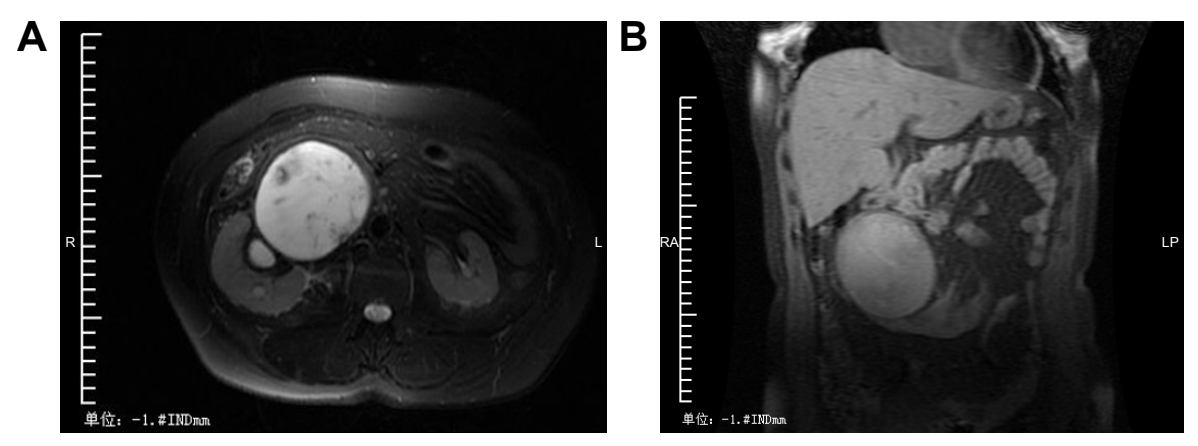

Figure 3 Magnetic resonance imaging of the abdomen, coronal view.

Notes: (A) Large unilocular hilar mass $(8 \times 7 \mathrm{~cm})$ in the right kidney with high signal intensity on $T_{2}$-weighted images. (B) The mass is isointense on $T_{1}$-weighted images. The horseshoe-kidney anomaly can also be observed.

demonstrated a horseshoe-kidney anomaly with a spherical cystic-solid mass $(9.5 \times 8.7 \mathrm{~cm})$, filling the proximal aspects of the right renal collecting system. CT angiography identified blood vessels supplying the tumor region (Figure 2C and D). Emission CT showed that the glomerular filtration rate was $33.1 \mathrm{~mL} / \mathrm{min}$ and $26.4 \mathrm{~mL} / \mathrm{min}$ in the right and left kidneys, respectively.

The patient underwent an isthmusectomy and a right nephrectomy. During exploratory laparotomy, a $10 \mathrm{~cm}$ large encapsulated mass containing approximately 2,000 mL thickly mucinous material was observed close to the renal hilum, and it was tightly adherent to the right kidney and the mesocolon with a free margin of a few millimeters from the normal renal pelvis. Laparotomy revealed that the appendix and both ovaries appeared macroscopically normal. Intraoperative frozen section biopsies from the cyst wall and intracystic papillary-like nodules pointed to a diagnosis of PMP (low grade). We speculated that the tumor was of renal origin, since its exact origin could not be defined.

A nephrectomy specimen $(14 \times 6 \times 5 \mathrm{~cm})$ with attached cystic tumor tissue was obtained. The postoperative pathological examination confirmed that the tumor was PMP, without evidence of renal parenchymal disease. The cystic mass $(8.5 \times 5 \times 3 \mathrm{~cm})$ extended from the hilum to the lower pole, with a wall of $0.2-0.5 \mathrm{~cm}$. The tumor capsule was gray-brown, while the inside wall was slightly rough and interrupted occasionally by cauliflower-like neoplasms. The cut surface showed myxoid changes with areas of fibrosis. On microscopic examination, pools of mucin were lined by malignant epithelium and areas of inflammation (Figure 4). Immunohistochemical staining results (Figure 5) suggested gastrointestinal origin $\left(\mathrm{CK} 20^{+}, \mathrm{CDX}_{2}^{+}\right.$, villin ${ }^{+}, \mathrm{CK}^{-}$, PAX8 ${ }^{-}, \mathrm{ER}^{-}, \mathrm{PR}^{-}, \mathrm{P}^{2} 3^{-}, \mathrm{Ki}^{+} 7^{+}$cells $50 \%$ ), indicating that the appendix was the most likely site of origin.

No adjuvant therapy was given to the patient after surgery. Ten months later, CEA and CA125 levels were $12.79 \mathrm{~g} / \mathrm{L}$ (normal $<5 \mathrm{~g} / \mathrm{L}$ ) and $9.1 \mathrm{kU} / \mathrm{L}$ (normal $<35 \mathrm{kU} / \mathrm{L}$ ), respectively. The patient has been in remission for 25 months and currently shows no clinical signs or CT evidence of disease recurrence or metastasis.

\section{Discussion}

With an estimated incidence of 1-2 persons per million per year, there has been considerable controversy on the nomenclature, pathology, origin, and treatment of PMP. ${ }^{3}$ In 1948, Bonann reported pseudomyxoma involving only the retroperitoneum. ${ }^{4}$ It has been reported that this disease is associated with genetic abnormalities, such as rearrangements of
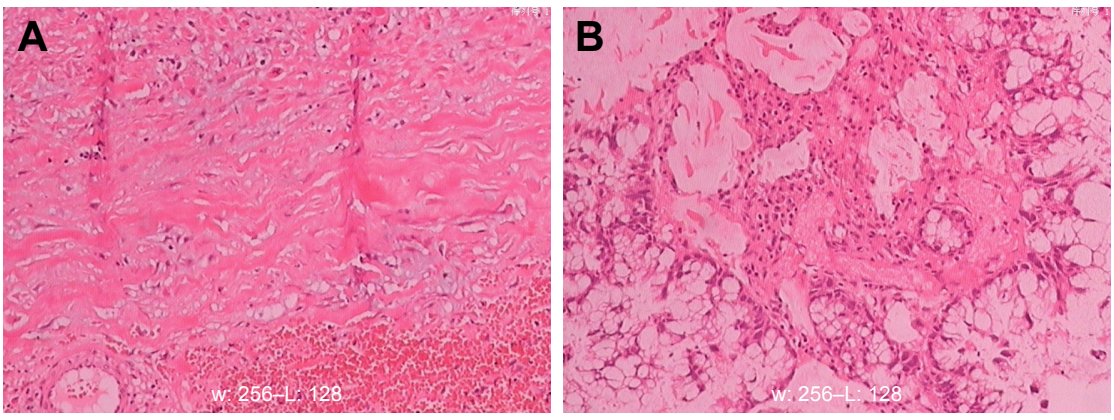

Figure 4 Histological examination of the tumor.

Notes: (A) Low-power view showing nests of tumor cells (H\&E, 200×). (B) Pools of mucin lined by malignant glandular epithelium with complex architecture in high-power view $(\mathrm{H} \& \mathrm{E}, 400 \times)$. 

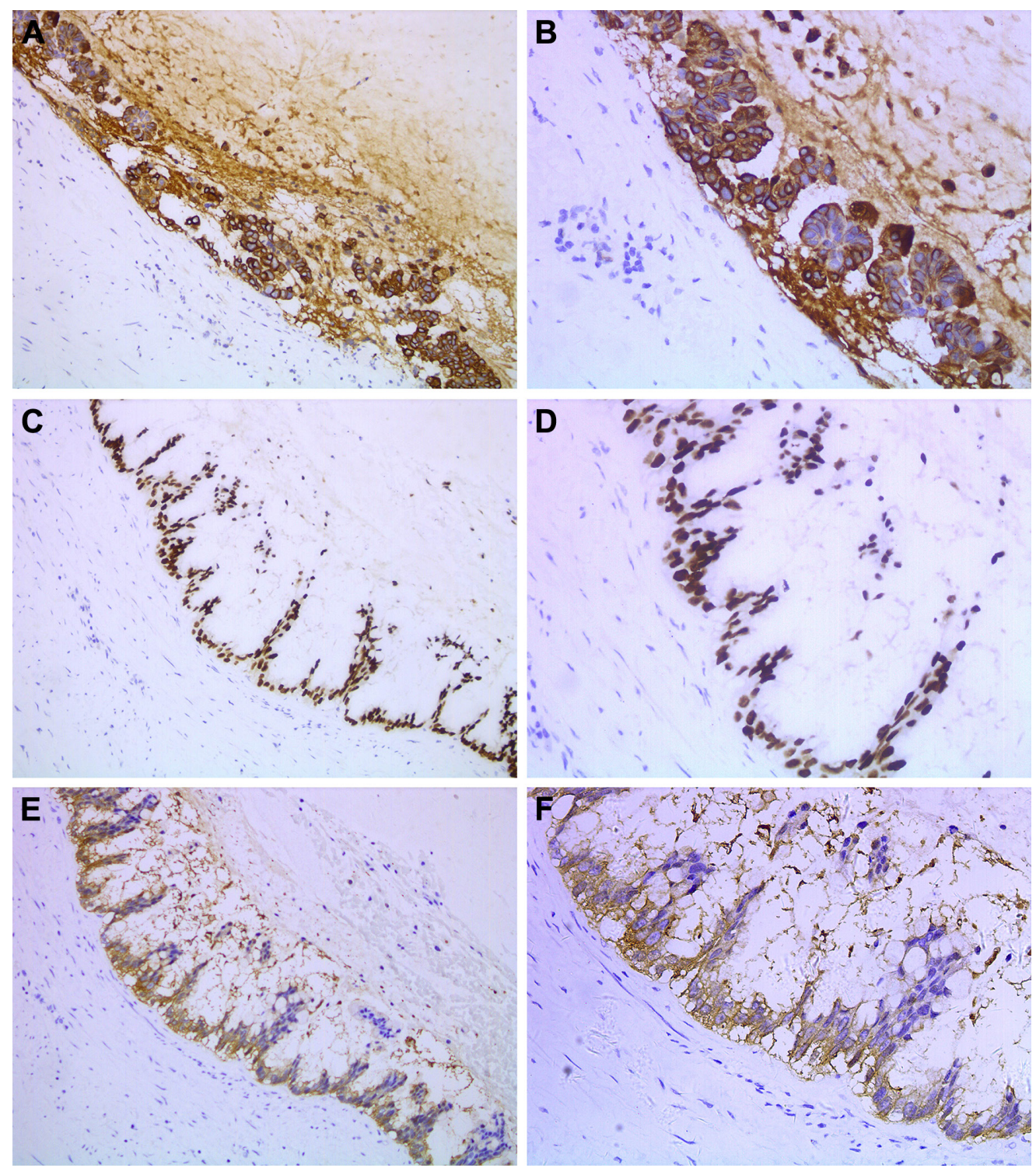

Figure 5 Immunohistochemical analysis showing that the tumor cells were positive for CK20.

Notes: (A) 200×; (B) 400×; (C) CDX2, 200×; (D) CDX2, 400×; (E) villin, 200×; (F) villin, 400×.

the KRAS gene and $M C L 1$ and $J U N$ gene amplification. ${ }^{5}$ This enigmatic disease presents a variety of aspecific and uncommon signs and symptoms, and routine laboratory tests are seldom conducive for diagnosis. ${ }^{6}$ As such, accurate diagnosis may be difficult before surgical corroboration. Previous literature reports have reported PMP presenting as an iliacus abscess, scrotal mass, or hydronephrotic kidney. ${ }^{7-9}$ In this case, an inflammatory response to mucin leads to fibrosis and encapsulation, limiting it to the retroperitoneum. When mucinous fluid-like materials localize in the renal hilum with renal involvement, this may mimic primary renal hilar tumor and be treated as a urological disease. There is insufficient research on PMP involving the kidneys to draw any firm conclusions regarding clinical manifestations, pathological features, or therapeutic approaches. We reviewed four other reports describing PMP affecting the kidneys and give the main findings in Table 1. ${ }^{9-12}$ In our case, however, the condition of the patient and tumor response were all inconsistent with those previously encountered. In the present case, the appendix appeared macroscopically normal, but according to the morphology of the epithelial lining and immunophenotype, the PMP seemed to be of appendiceal origin.

The pathological classification and biological behavior of PMP has been controversial for many years. There is considerable variability in the criteria and appellations used by different pathologists to diagnose PMP. Ronnett et a ${ }^{13}$ suggested a classification based on tumor pathology, where they placed all PMP into three groups: disseminated peritoneal adenomucinosis (DPAM), peritoneal mucinous carcinoma (PMCA), and peritoneal mucinous carcinoma with 


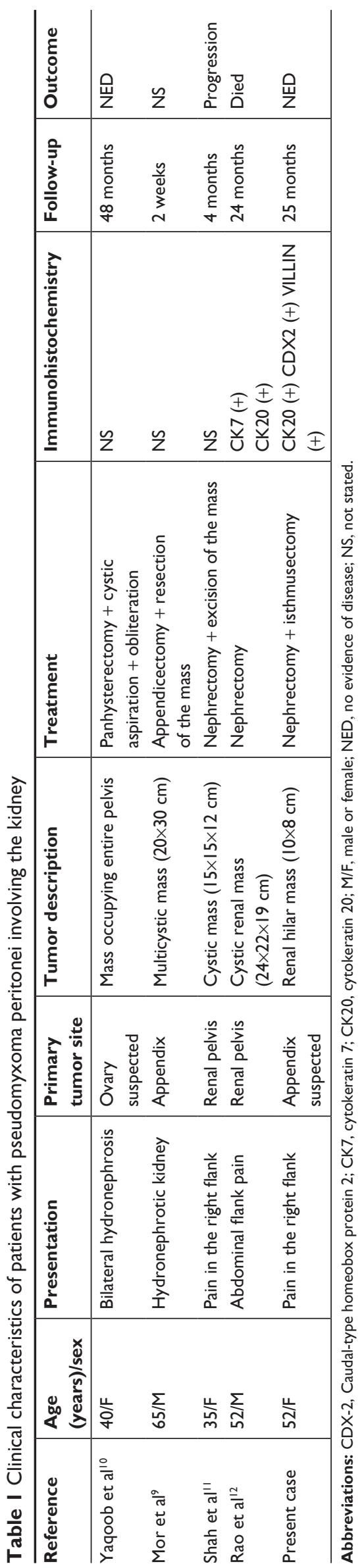

intermediate or discordant features (PMCA-I/D). According to this prevailing method for PMP, DPAM includes histologically benign peritoneal lesions associated with ruptured appendiceal mucinous adenomas, as well as those with similar pathology but lacking a demonstrable appendiceal adenoma. Fortunately, consensus was achieved on the pathologic classification of PMP at the 2012 World Congress of the Peritoneal Surface Oncology Group International. Three categories of PMP were accepted - low grade, high grade, and high grade - with signet-ring cells. For cases in which epithelial cells were not found, "acellular mucin" was the term used. It was agreed that low-grade and high-grade mucinous carcinoma peritonei should be considered synonymous with DPAM and PMCA, respectively..$^{14}$ This case should be classified as PMP with low-grade histological features.

PMP has traditionally been considered a "borderline" malignancy. Despite their low malignant potential, PMP neoplasms are heterogeneous. Their biological behavior can be highly variable, and up to $30 \%$ of patients will die as a result of progressive disease. ${ }^{2}$ The most important single factor determining survival is the ability to achieve complete tumor removal. On our surgical exploration, the cystic-solid tumor was found to be in the vicinity of the right renal hilum with a free margin of a few millimeters from the normal renal pelvis.

Macroscopically, there was no necrotic appendix or ovaries, and no duodenum or colon invasion was found. After a multidisciplinary council, the risks and benefits of surgery were discussed, and she underwent an isthmusectomy and right nephrectomy. The shortcoming of this case report is that the appendectomy has not been performed, due to scarcity of experience. It has been proffered that traditional surgery involving repeated debulking is associated with a limited expectation of long-term survival, and that only $15 \%$ of patients treated by surgery alone can survive longer than 5 years. ${ }^{1}$ Cytoreductive surgery, with complete cytoreduction whenever possible, combined with hyperthermic intraperitoneal chemotherapy, appears to improve overall survival to $91 \%$ at 5 years. ${ }^{15}$ Chua et a ${ }^{16}$ investigated the impact of combining aggressive debulking surgery with intraperitoneal chemotherapy. Compared to patients treated by surgery alone, patients subjected to cytoreduction of minimal peritoneal surface residual lesions and adjuvant intraperitoneal chemotherapy indicated better survival rates. The prognosis of PME is likely to be better than PMP, as vital abdominal structures are not involved. Furthermore, new research shows that mucin directs the biology of the PMP tumor, especially Muc2, which could serve as a molecular marker for PMP. ${ }^{3}$ The development of mucin-targeted therapies could be a promising avenue for future research. 
Some tumor markers have been documented to be valuable in the management of PMP - CEA, CA19.9, and CA125 - albeit these are relatively aspecific. ${ }^{17}$ Elevated levels of tumor markers indicate more aggressive disease, with increased risk of recurrence, progression, and poorer prognosis. This patient has refused postoperative further treatment. After a systematic follow-up and radiological review, there was no luciferous evidence at the appendix and localized renal area to indicate the onset of recurrence or metastasis, despite persistently elevated CEA levels on follow-up. However, further follow-up will be required, due to the unknown clinicopathological properties of the appendix.

\section{Conclusion}

PMP that both localizes in the retroperitoneum and involving the urinary system is rather rare. Accurate and definite diagnosis of such is difficult due to aspecific clinical presentations. Herein, we report an exceptionally rare case in which a PME is presented as a simple renal hilar mass associated with horseshoe kidney, mimicking a tumor of renal origin. Our experience also underlines the importance of involving surgeons in the investigation and the management of atypical retroperitoneal masses.

\section{Ethics statement}

This case report was approved by the Medical Ethics Committee of the Second Affiliated Hospital of Dalian Medical University. Written informed consent was obtained from the patient for publication of this case report and accompanying images.

\section{Acknowledgments}

This study was supported by grants from the Natural Science Foundation of China (81772739) and the Natural Science Foundation of Liaoning Province (No. LQ2017025). The authors thank Daqing Xie, MD and Gerard Nkengurutse, MD for their contribution to the review and revision of the manuscript.

\section{Disclosure}

The authors report no conflicts of interest in this work.

\section{References}

1. Amini A, Masoumi-Moghaddam S, Ehteda A, Morris DL. Secreted mucins in pseudomyxoma peritonei: pathophysiological significance and potential therapeutic prospects. Orphanet J Rare Dis. 2014;9:71.

2. Baratti D, Kusamura S, Milione M, et al. Pseudomyxoma peritonei of extra-appendiceal origin: a comparative study. Ann Surg Oncol. 2016; 23(13):4222-4230.

3. Nakakura EK. Pseudomyxoma peritonei: more questions than answers. $J$ Clin Oncol. 2012;30(20):2429-2430.

4. Bonann LJ, Davis JG. Retroperitoneal mucocele of the appendix; a case report with characteristic roentgen features. Radiology. 1948; 51(3):375-382.

5. Pietrantonio F, Perrone F, Mennitto A, et al. Toward the molecular dissection of peritoneal pseudomyxoma. Ann Oncol. 2016;27(11): 2097-2103.

6. Smeenk RM, van Velthuysen ML, Verwaal VJ, Zoetmulder FA. Appendiceal neoplasms and pseudomyxoma peritonei: a population based study. Eur J Surg Oncol. 2008;34(2):196-201.

7. Edrees WK, Hannon RJ. Pseudomyxoma extraperitonei: a rare presentation mimicking an iliacus abscess. Ulster Med J. 1999;68(1):38-39.

8. Baker WC, Goldman LB, deVere White RW. Pseudomyxoma peritonei presenting as a scrotal mass. J Urol. 1988;139(4):821-822.

9. Mor Y, Leibovitch I, Garniek A, Avigad I, Jonas P, Goldwasser B. Case report: pseudomyxoma extraperitonei: an unusual presentation mimicking a hugh hydronephrotic kidney. Clin Radiol. 1996;51(3):221-222.

10. Yaqoob M, Fahal IH, Finn R. Obstructive nephropathy due to pseudomyxoma peritonei and its management by cystic obliteration and urinary diversion. Nephrol Dial Transplant. 1991;6(1):60-61.

11. Shah VB, Amonkar GP, Deshpande JR, Bhalekar H. Mucinous adenocarcinoma of the renal pelvis with pseudomyxoma peritonei. Indian $J$ Pathol Microbiol. 2008;51(4):536-537.

12. Rao P, Pinheiro N Jr, Franco M, et al. Pseudomyxoma peritonei associated with primary mucinous borderline tumor of the renal pelvicalyceal system. Arch Pathol Lab Med. 2009;133(9):1472-1476.

13. Ronnett BM, Zahn CM, Kurman RJ, Kass ME, Sugarbaker PH, Shmookler BM. Disseminated peritoneal adenomucinosis and peritoneal mucinous carcinomatosis. A clinicopathologic analysis of 109 cases with emphasis on distinguishing pathologic features, site of origin, prognosis, and relationship to "pseudomyxoma peritonei". Am J Surg Pathol. 1995;19(12):1390-1408.

14. Carr NJ, Cecil TD, Mohamed F, et al; Peritoneal Surface Oncology Group International. A consensus for classification and pathologic reporting of pseudomyxoma peritonei and associated appendiceal neoplasia: The results of the peritoneal surface oncology group international (PSOGI) modified delphi process. Am J Surg Pathol. 2016;40(1):14-26.

15. Sugarbaker PH. New standard of care for appendiceal epithelial neoplasms and pseudomyxoma peritonei syndrome? Lancet Oncol. 2006;7(1):69-76.

16. Chua TC, Moran BJ, Sugarbaker PH, et al. Early- and long-term outcome data of patients with pseudomyxoma peritonei from appendiceal origin treated by a strategy of cytoreductive surgery and hyperthermic intraperitoneal chemotherapy. J Clin Oncol. 2012;30(20):2449-2456.

17. Nummela $P$, Leinonen $H$, Järvinen $P$, et al. Expression of CEA, CA19-9, CA125, and EpCAM in pseudomyxoma peritonei. Hum Pathol. 2016; $54: 47-54$.

\section{Dovepress}

\section{Publish your work in this journal}

OncoTargets and Therapy is an international, peer-reviewed, open access journal focusing on the pathological basis of all cancers, potential targets for therapy and treatment protocols employed to improve the management of cancer patients. The journal also focuses on the impact of management programs and new therapeutic agents and protocols on

Submit your manuscript here: http://www.dovepress.com/oncotargets-and-therapy-journal

patient perspectives such as quality of life, adherence and satisfaction. The manuscript management system is completely online and includes a very quick and fair peer-review system, which is all easy to use. Visit http://www.dovepress.com/testimonials.php to read real quotes from published authors. 